\title{
High-Contrast Imaging of Nanodiamonds in Cells by Energy Filtered and Correlative Light-Electron Microscopy: Towards a Quantitative Nanoparticle-Cell Analysis
}

Shen Han, ${ }^{1}$ Marco Raabe, ${ }^{1,2}$ Lorna Hodgson, ${ }^{3}$ Judith Mantell, ${ }^{3}$ Paul Verkade, ${ }^{3}$ Theo Lasser, ${ }^{1,4}$ Katharina Landfester, ${ }^{1}$ Tanja Weil, ${ }^{*}, 1,2$ and Ingo Lieberwirth, ${ }^{*}, 1$

1. Max-Planck Institute for Polymer Research, Mainz, Germany.

2. Institute of Inorganic Chemistry I, Ulm University, Ulm, Germany.

3. School of Biochemistry, University of Bristol, Bristol, UK.

4. Laboratoire d'Optique Biomédical, École Polytechnique Fédérale de Lausanne, Lausanne, Switzerland.

* Corresponding author: hanshen@mpip-mainz.mpg.de

During the last decades, nanoparticles have shown great potential as drug carriers and probes for bioimaging. However, on one hand, classical light microscopy (LM) is limited by the wavelength of light and does not provide resolution beyond the diffraction limit $(\sim 200 \mathrm{~nm})$ [1]. On the other hand, electron microscopy (EM) provides morphological visualization at the low nanometer scale, however, such studies are challenging to conduct due to the low contrast of single nanoparticles to the surrounding resin, and EM only generates grayscale images. Correlative light and electron microscopy (CLEM) is designated to overcome the hurdles of both microscopies. By obtaining fluorescence signal of the sample in LM and sequentially image the same region of interests (ROIs) in EM, labeled biomolecules as well as nanoparticles are able to be localized in high resolution EM image together with their fluorescence.

Fluorescent nanodiamonds (fNDs) have emerged as powerful imaging probes in LM, as drug transporter and for nanoscale-sensing applications [2]. fNDs are carbon-based nanoparticles with nitrogen vacancy (NV) defect centers that provide unconditionally stable fluorescence without blinking ideal for singleparticle tracking [3] and super-resolution studies in vitro [4].

In this study, we demonstrate a stringent procedure to image and quantify coated fNDs as clusters as well as down to the single particle level inside cells. fNDs are proposed as emerging nanomaterial for CLEM due to their consistent photostability, which provides significant advantages compared to the weak fluorescence of gold nanoparticles [5] and blinking problems of QDs [6]. We could image fNDs at different stages during their cellular uptake and intracellular trafficking highlighting that many cellular barriers have to be crossed in drug delivery. fND clusters appeared outside the cellular membrane as well as in endosomal vesicles as detected by CLEM with a high contrast by colocalization of LM and EM signals (Figure 1c). EM images of fND clusters were obtained with the highest resolution reported yet, which even allowed the quantification of individual fNDs in the larger fND clusters inside intracellular vesicles. Furthermore, individual fNDs were detected for the first time by EFTEM inside a newly forming, early endosomal vesicle during the cellular uptake process (Figure 1e) as well as in a mitochondrion (Figure 1d).

References:

[1] L Schermelleh et al., Nat Methods 2 (2005), p. 743.

[2] M Chipaux et al., Small 14 (2018), p. e1704263. 
[3] CC Fu et al., Natl Acad Sci USA 104 (2007), p. 727.

[4] S Arroyo-Camejo et al., Acs Nano 7 (2013), p. 10912.

[5] H He et al., Anal Chem 80 (2008), p. 5951.

[6] S Bianco et al., The Journal of Chemical Physics 123 (2005), p. 174704.

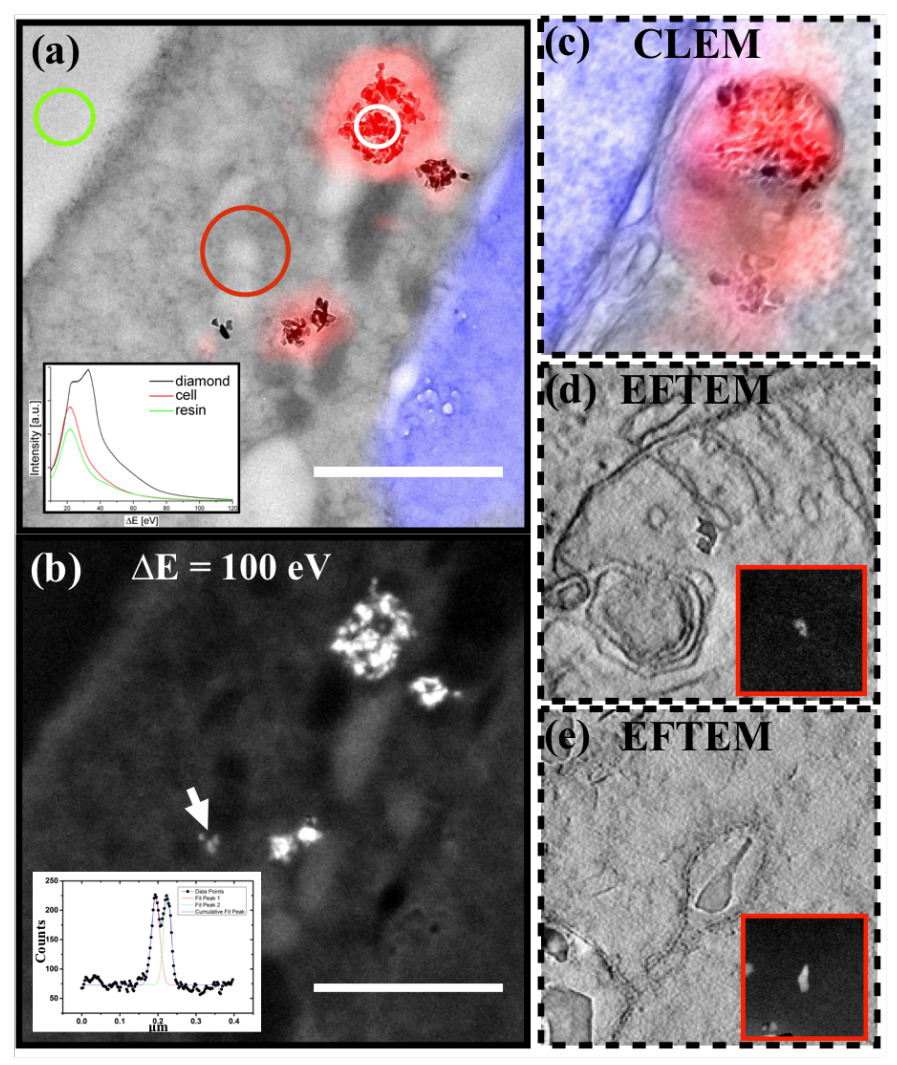

Figure 1. Single fND and fND clusters imaged by correlative light-electron microscopy (CLEM) and energy filtered transmission electron microscopy (EFTEM). (a) CLEM overlay (inset: EEL spectra of embedded fNDs (black), the embedded cell (red) and a resin-only area (green), corresponding to the positions indicated in (a)); (b) EFTEM micrograph acquired at an energy loss of $100 \mathrm{eV}$ with $10 \mathrm{eV}$ slit width. The white arrows indicate the detection of individual fNDs. (inset: Gaussian line profile value of two closely located fNDs); (c) Identification and quantification of fNDs in intracellular vesicles using CLEM. In addition, single fND detection was achieved by EFTEM (d) inside a mitochondrion as well as (e) in the cellular membrane during fND uptake. Scale bar: $1 \mu \mathrm{m}$. 